\title{
A 1 GHz Oscillator-Type Active Antenna
}

\author{
Jennifer L. Jordan*, Maximilian Scardelletti, and George E. Ponchak \\ The NASA Glenn Research Center, Cleveland, OH, 44135 \\ E-mail: Jennifer.L.Jordan@nasa.gov
}

\section{Introduction}

Wireless sensors are desired for monitoring aircraft engines, automotive engines, industrial machinery, and many other applications. The most important requirement of sensors is that they do not interfere with the environment that they are monitoring. Therefore, wireless sensors must be small, which demands a high level of integration. Sensors that modulate an oscillator active antenna have advantages of small size, high level of integration, and lower packaging cost.

Several types of oscillator active antennas have been reported. Ip et al. demonstrated a CPW line fed patch antenna with a feedback loop [1]. No degradation in performance was noticed without a ground plane. A GaAs FET was used in an amplifier/oscillator-based active antenna [2]. An oscillator based on a Cree $\mathrm{SiC}$ transistor was designed and characterized in [3]. This paper reports the integration of the SiC Clapp oscillator to a slotline loop antenna.

\section{Design and Fabrication}

A square, slotline, loop antenna designed to radiate at $1 \mathrm{GHz}$ was fabricated on a 20 mil thick, 2 inch square $99.6 \%$ polycrystalline alumina substrate with a relative dielectric constant $\varepsilon_{\mathrm{r}}$ of 9.7 . The mean free path of the antenna is approximately one wavelength at $1 \mathrm{GHz}$, and Ansoft HFSS electromagnetic simulator is used to optimize the antenna dimensions. A $\mathrm{Cr} / \mathrm{Au}$ metal layer 50/2500 nm thick was deposited and lithographically patterned to develop the antenna. There is no ground plane on the backside of the substrate. Figure 1 shows a microphotograph of the antenna with dimensions in millimeters.

Figure 2a is a schematic of the Clapp oscillator discussed in [3]. The $1 \mathrm{GHz}$ Clapp oscillator was epoxied and wire bonded to the antenna. The Clapp oscillator consists of a Cree $\mathrm{SiC}$ MESFET transistor, multilayer chip capacitor $\left(\mathrm{C}_{1}, \mathrm{C}_{2}\right.$, and $\mathrm{C}_{\mathrm{T}}$ are 4.0, 4.0, and $10.0 \mathrm{pF}$, respectively) and a spiral inductor $\left(\mathrm{L}_{\mathrm{T}}=8.5 \mathrm{nH}\right)$. Figure $2 \mathrm{~b}$ shows the fabricated oscillator integrated with the antenna. The two probe pads in Fig. $2 \mathrm{~b}$ are used for providing the drain voltage and for measuring the direct power from the oscillator. $\mathrm{V}_{\mathrm{GS}}$ is applied by a needle probe directly to the bias pad. Single wire bonds are used for the DC connections. A pair of wire bonds is used for connecting the RF output of the oscillator to the inner and outer edge of the slotline loop. The pair of wire bonds have less loss than a single wire bond, which minimizes the degradation of the oscillator performance. 


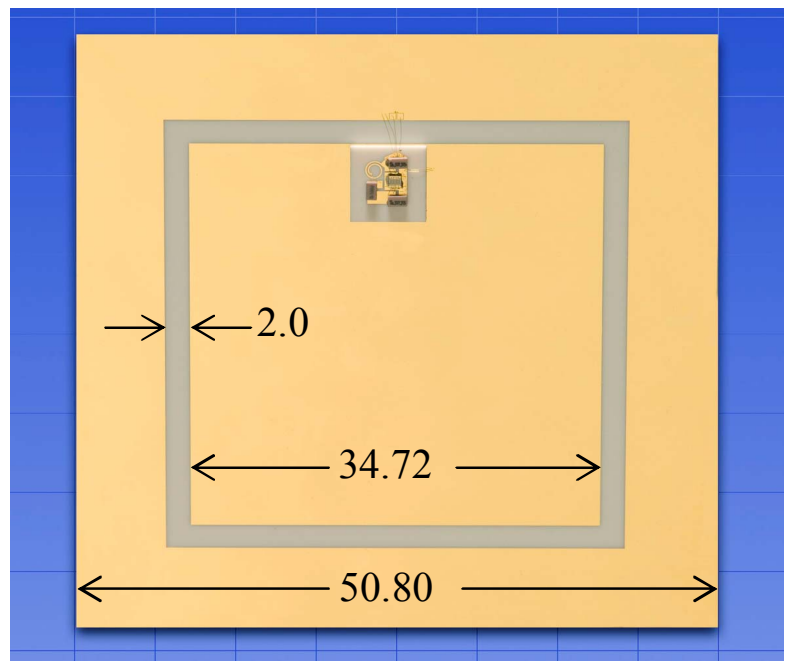

Figure 1: Microphotograph of active antenna with dimensions in millimeters
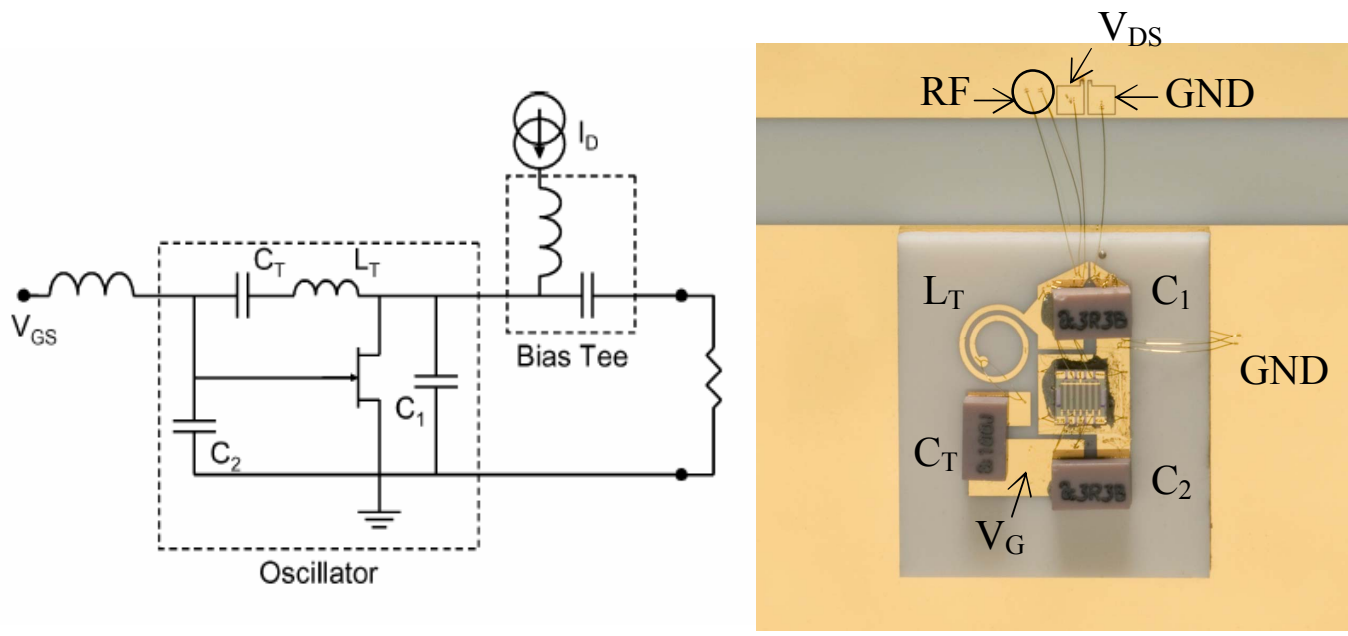

Figure 2: (a) Clapp oscillator schematic and (b) microphotograph

\section{Antenna and Oscillator Characterization}

First, the antenna is characterized before the oscillator is integrated by using the ground-signal probe pads seen in Fig. 2b. A pair of bond wires is used to connect the two probe pads to the inner and outer edges of the slotline. Using an RF probe station, the planar antenna was placed on a ceramic chuck and characterized with an HP 8510C VNA. The reflection measurement is shown in Fig. 3 with a resonant frequency of $1.0685 \mathrm{GHz}$. Since there is no ground plane, some radiation may penetrate down into the ceramic block. 


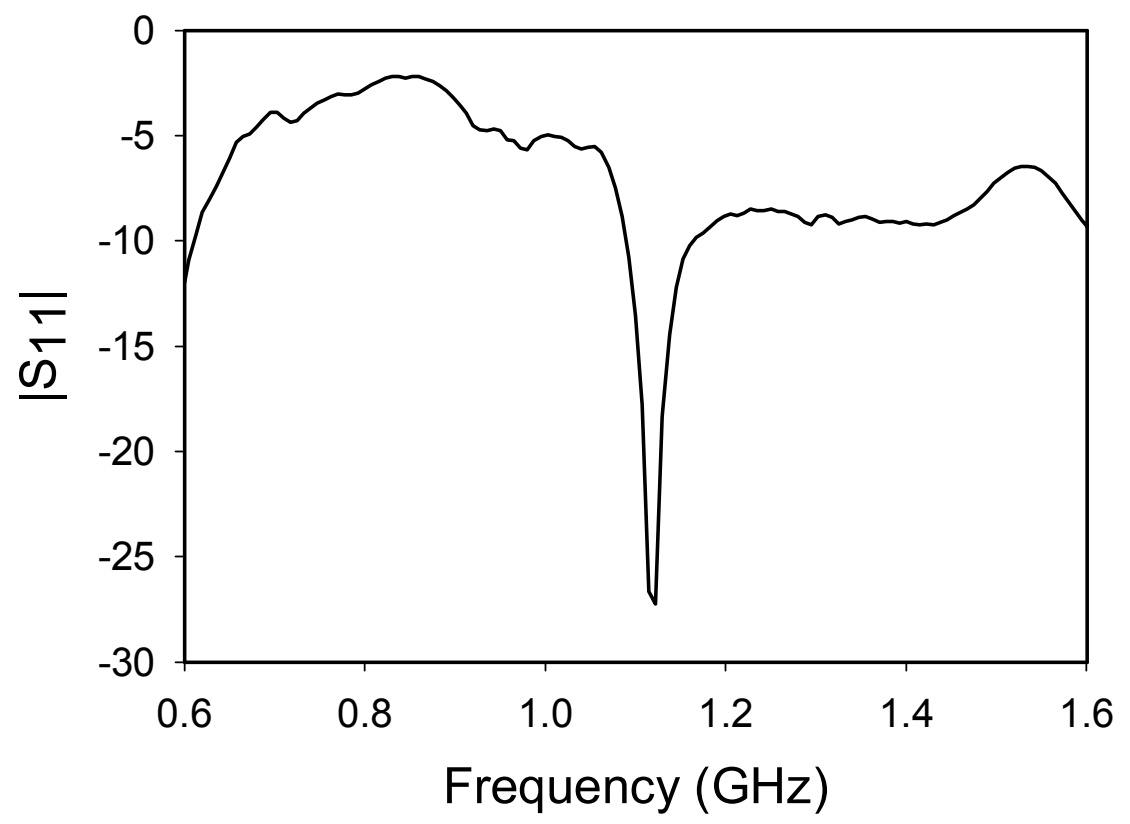

Figure 3: Planar antenna $S_{11}$ reflection measurement

The active antenna is characterized using the same RF probe station, ceramic chuck, and two Agilent E4448A PSA Series Spectrum Analyzers. A groundsignal probe and a bias-T are used to provide bias to the oscillator through the probe pads. To measure the radiated power, a dipole antenna soldered to semirigid coax and tuned for $1 \mathrm{GHz}$ is placed $50 \mathrm{~cm}$ directly above the active antenna. The two spectrum analyzers, one connected to the dipole antenna and the other to the RF probe, are used to measure the radiated and direct spectrum, respectively. The transistor is biased with $\mathrm{V}_{\mathrm{DS}}=10 \mathrm{~V}$ and $\mathrm{I}_{\mathrm{DS}}=100 \mathrm{~mA}$.

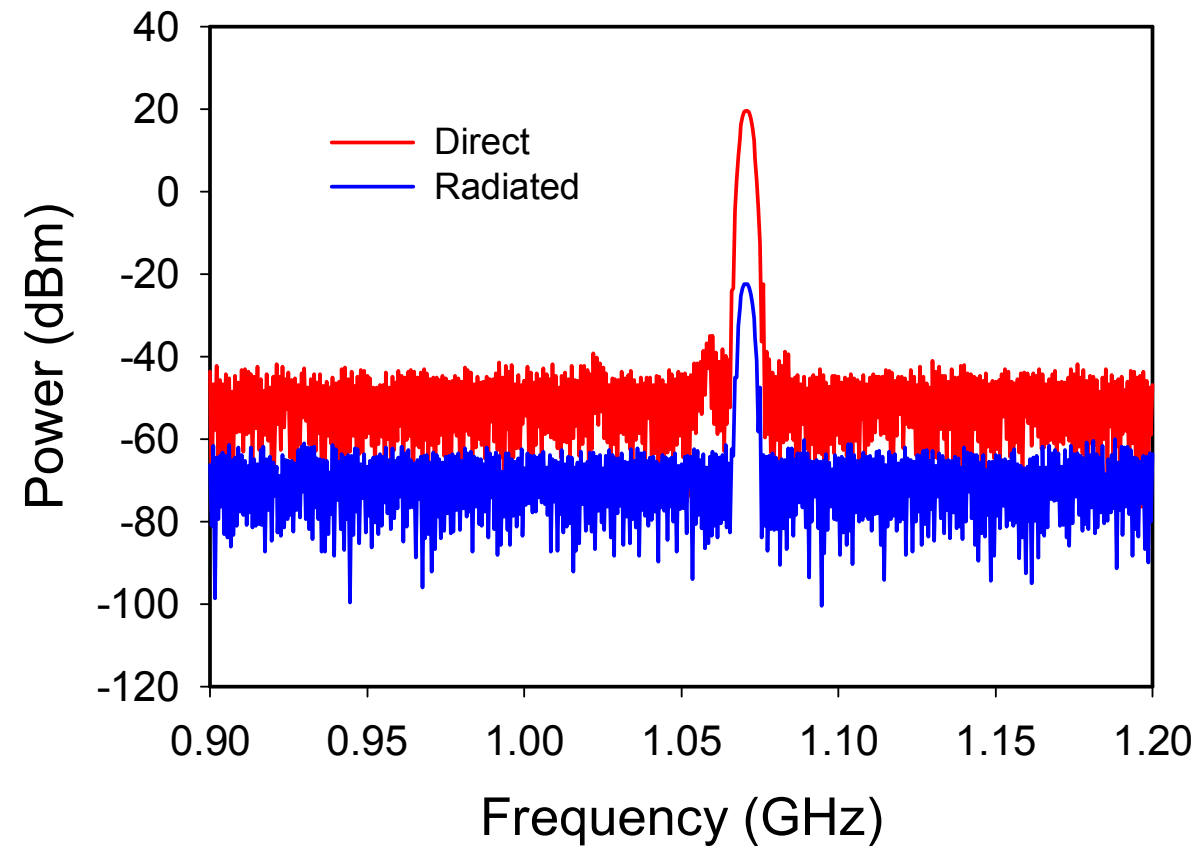

Figure 4: Power spectrum for direct and coupled antenna 
The direct measured spectrum (at the probe pads) of the active antenna and the radiated signal measured by the dipole antenna are shown in Fig. 4. The transmitting and receiving antennas coupled at $1.0705 \mathrm{GHz}$, varying slightly from the $\mathrm{S}_{11}$ resonance of the slotline antenna. The measured power at the probe pads is $19.74 \mathrm{dBm}$ and the measured power at the dipole is $-22.29 \mathrm{dBm}$. Note that no corrections for cable and bias-T losses have been made. Also, the power measured at the probe pads (direct power) is not the power transmitted by the active antenna.

\section{Conclusion}

An oscillator-type active antenna has been built and characterized. The oscillator frequency has been shown to not be affected by the antenna loading. This circuit will form the basis for a high temperature wireless sensor.

\section{Acknowledgments}

The authors would like to thank Nick Varaljay and Elizabeth McQuaid for their fabrication efforts. This work was supported by the Wireless Communications group of the Integrated Vehicle Health Management (IVHM) project at NASA.

\section{References:}

[1] K.H.Y. Ip and G.V. Eleftheriades, "A compact CPW-based single-layer injection-locked active antenna for array applications," IEEE Transactions on Microwave Theory and Techniques, Vol. 50, Issue 2, pp. 481 - 486, Feb. 2002.

[2] I. Waldron, A. Ahmed and S. Makarov, "Amplifier-based active antenna oscillator design at 0.9-1.8 GHz." IEEE/ACES Int. Conf. on Wireless Communications and Applied Computational Electromagnetics, April 3-7, 2005, pp. $775-778$.

[3] Z. D. Schwartz and G. E. Ponchak, "1 GHz, $200{ }^{\circ} \mathrm{C}$, SiC MESFET Clapp oscillator," IEEE Microwave and Wireless Component Lett., Vol. 15, No. 11, pp. 730-732, Nov. 2005. 